\title{
Seamless User Notification in Ambient Soundscapes
}

\author{
Andreas Butz \\ University of Munich (LMU) \\ LFE Media Informatics \\ Amalienstrasse 17 \\ 80333 Munich, Germany \\ butz@ifi.Imu.de
}

\author{
Ralf Jung \\ Saarland University \\ Computer Science \\ Stuhlsatzenhausweg, Bldg. 36.1 \\ 66123 Saarbrücken, Germany \\ rjung@xantippe.cs.uni-sb.de
}

\begin{abstract}
We describe a method for notifying users through auditory cues embedded in an ambient soundscape in the environment. It uses pieces of music which are composed in such a way, that particular instruments or motifs can be added or omitted without losing the aesthetic quality of the overall composition. This allows for very subtle modifications in the soundscape which are only noticed by those users who have chosen this particular instrument or motif as "their" notification instrument before. As a side effect, the soundscape itself can be used to subtly influence the mood of users. The method has been implemented in a prototype, which we briefly discuss. The prototype is implemented using a spatial audio framework and can hence notify users from particular directions.
\end{abstract}

\section{Categories and Subject Descriptors}

H.5.2 [User Interfaces]: Auditory (non-speech) feedback; H.5.2 [User Interfaces]: Theory and methods; H.5.2 [User Interfaces]: Evaluation/methodology

\section{General Terms}

Design, Human Factors, Experimentation, Theory

\section{Keywords}

Ambient Audio, Soundscapes, Auditory Cues, Peripheral Awareness

\section{INTRODUCTION}

The auditory sense is still mostly neglected in today's user interfaces. Graphical interfaces use simple pieces of sound in order to provide feedback for interaction or to notify users of certain events, such as errors, warnings or successful execution of a command. Even if these sounds adhere to a common theme, they still stand for themselves and are neither integrated into a coherent soundscape or composition, nor

Copyright is held by the author/owner. IUI'05, January 10-13, 2005, San Diego, California, USA. ACM 1-58113-894-6/05/0001. coordinated with other sounds present in the environment. In the vision of ubiquitous computing, the user interfaces are part of our everyday environments. For the acoustic part of the interface this means, that it has to be integrated into or coordinated with existing sounds. Already today, music is used in shopping malls to subtly influence customers, lift their mood, and hence increase consumer spending. The music used in these environments, snidely called "elevator music", is perceived only peripherally. If the background music plays a tune which we particularly like or dislike, it often crosses the border from peripheral to conscious perception and we suddenly become aware of it.

In our research, we try to create this same effect by providing a permanent soundscape which eventually vanishes into peripheral perception, but comes back to consciousness only for a particular user, as soon as it contains certain elements which this user has been trained to observe beforehand.

\section{RELATED WORK}

Kilander and Lönnqvist have used natural sounds in their work on weakly intrusive ambient soundscapes [2]. These sounds blend into the natural noise of the surroundings and the degree of their intrusiveness can be regulated. They are, however, chosen arbitrarily and don't belong to a common theme, metaphor or composition. In our work we use notification sounds integrated into composed music. Mynatt et al. used voice, music, sound effects or a combination of these as notification sounds in their audio aura system[3]. In terms of music they used different short melodies carrying different meanings. They were not integrated into a comprehensive composition playing continuously.

The composition and arrangement of musical patterns and songs is the only way to ensure full control of all music factors. Different methods have been used for automatic composition of music, such as grammars, finite state automata or constraints. Roads [4] provides an overview of these techniques. Zimmermann[1] developed a system for automated music composition in order to assist multi media presentations. In our research we use music composed by humans, but let the system determine the mix of different pre-produced parts.

\section{SOUNDSCAPES WITH OPTIONAL ELEMENTS}

The soundscapes used in our approach are musical compositions with a fixed core and a certain number of optional elements. The core provides a coherent musical structure in 
terms of rhythm, melody and harmony. Optional elements can be either additional instruments which are not present in the core part, or additional musical elements, such as a new melodic motif or rhythmic pattern.

One of these optional elements is chosen by the user beforehand. Users can, for example, specify the snare drum as "their instrument" or a specific rhythmic pattern as "their riff $^{1 "}$. The compositions are normally played in the environment without these optional elements. In order to get the attention of a specific user, the corresponding optional element is added to the soundscape. The user who has chosen or learnt this element before, will notice the change and realize that it is meant as a notification for him, while other users won't be able to distinguish the optional element from the core.

In order to test this method, we have arranged two own compositions and a Jazz standard in such a way, that they sound "complete" if only the musical core is played, but that additional elements can be added without overloading the composition. Particular care had to be taken about the volume, the rhythmic and the melodic properties of all parts, in order to ensure that the optional parts are neither missed when they are absent, nor disturb when they are present.

The overall nature of the arrangements should be modest and unobtrusive, so that the music can be played in the background without disturbing. It should be calm and serene in order to lift people's moods as a side effect. To meet these requirements, we used simple moderate melody patterns, which appear in different instruments and alterations to prevent the effect of a catchy tune. We also left out text and human voices altogether, because they easily catch people's attention and text usually also carries a meaning, which might be misleading in our context. As rhythm and melody instruments, we use guitars, piano and flutes with melodical riffs no shorter than 16 bars to prevent memory effects.

For the ambient musical carpets we use keyboards with string sounds and prefer "sus2" 2 chords to create a warm and unobtrusive sound. The speed of songs ranges between 60 and 80 beats per minute, which is similar to the normal human heart frequency and has a calming effect on the body. The use of sporadic and moderate drum patterns assists this calming effect. Finally, we avoided big pitch jumps in the melody instruments and handled their volume with care. All instruments are natural instruments and were recorded via microphones (drum and piano patterns), magnetic and piezo pickups (bass, guitars) and the use of $\mathrm{MIDI}^{3}$ (keyboards).

\section{PROTOTYPE AND USER STUDY ENVIRONMENT}

Figure 1 shows the structure of our implemented prototype system. Compositions are stored in a sound repository as multi channel audio files. They contain a track with the core part and one track for each optional part. In our test scenario the user chooses which songs are played and which optional part is used as her personal notification part.

\footnotetext{
${ }^{1}$ a musical riff is a short, repeated pattern of music which can be played throughout the piece

${ }^{2}$ musical chord description where the third of the triad is replaced by the second tone

${ }^{3}$ Musical Instrument Digital Interface
}

The sound files each create an audio object in the $\mathrm{SAFIR}^{4}$ framework[5], which mixes them together and plays the result over currently eight loudspeakers in our instrumented environment.

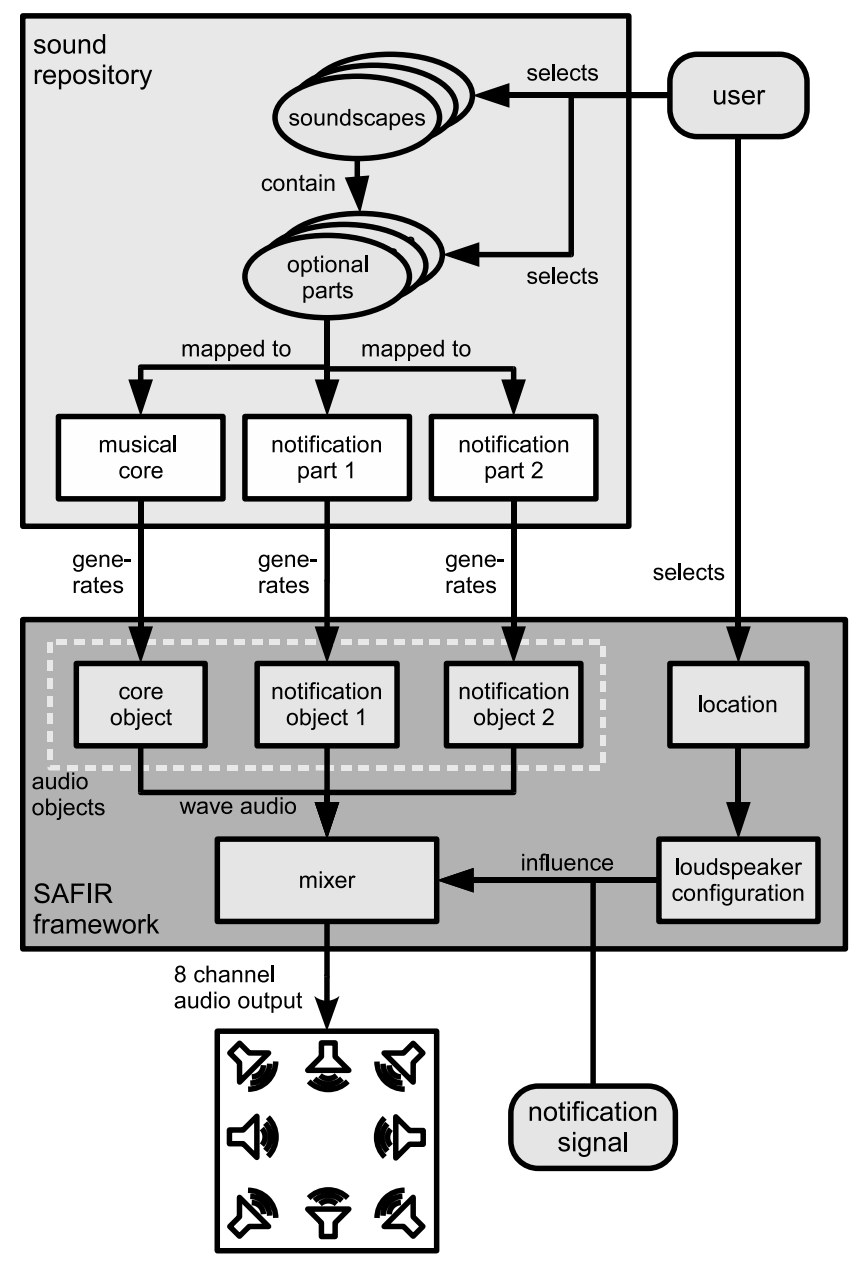

Figure 1: Structure of our prototype system

When the system starts, the audio mix only contains the core part of the composition. If the external notification signal for a user is triggered, the user's particular notification sound is added to the mix at the next musically possible point in time. This means, for example, that rhythmic patterns are only added at full bars, and optional motifs are only played where they make harmonic sense. Depending on the user's location, a notification can also be given from a particular direction. Thereby, the user will not only notice the notification, but also where it came from. The environment can thereby make the notification appear as if it came from a particular object or device. Other users will remain undisturbed, since they have chosen different notification sounds and won't be able to distinguish the optional element from the core. In order to test our approach in a user study, we have written a simple graphical interface (see Figure 2), in which the user can choose one of the recorded soundscapes, as well as the notification instrument or pat-

\footnotetext{
${ }^{4}$ Spatial Audio Framework for Instrumented Rooms
} 


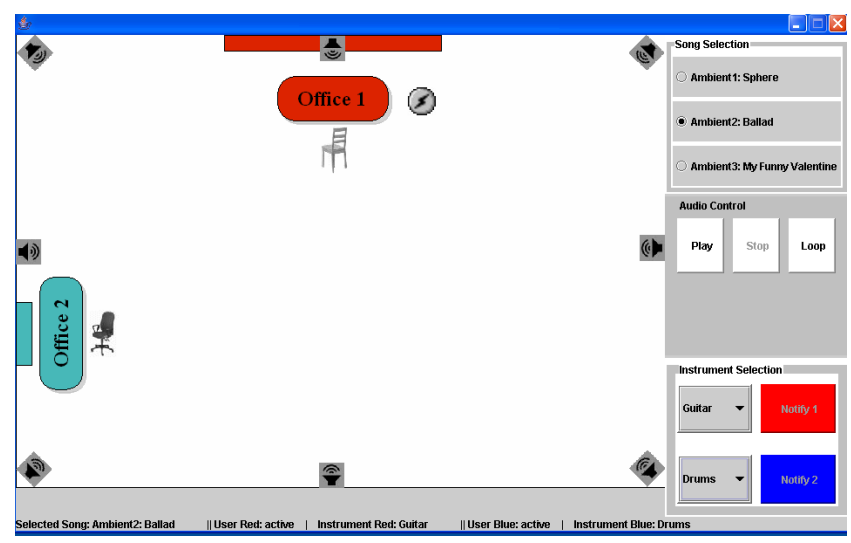

Figure 2: Graphical interface for the test scenario

tern. After starting the core sound, the system can receive the notification command and will add the corresponding sound to the mix at the next possible point in time.

\section{SOME LIMITATIONS AND POTENTIAL APPLICATIONS}

Due to the nature of our approach, it doesn't scale well to many different users with independent notification sounds, since this would demand very complex compositions with many optional tracks. Since the notification sounds are only played when they make musical sense, which might (in the worst case) only be 15 bars later, the approach is unsuitable for time critical applications. Finally, our approach requires compositions which are structured, recorded and produced with optional parts around a musical core. While this would mean substantial effort and cost in regular music productions, it might be more feasible in fully electronic (MIDI-based) productions, which in turn can often be found in today's low cost "elevator music".

We see the primary application of our method in instrumented environments with only a few users, where it is necessary to notify one user without disturbing others or disrupting the existing atmosphere. Examples of such environments are workplaces, stores or shopping malls, where ambient music is already used today, but also museums or memorial places, where a particular atmosphere has to be kept and alarm sounds or traditional announcements are out of the question. In these environments, notifications for a limited set of employees could be seamlessly integrated into the existing soundscape.

\section{CONCLUSIONS AND FUTURE WORK}

We have presented an approach for the acoustic notification of users in ambient soundscapes. The main contribution of our research is that we can provide notifications to one user while others remain undisturbed, and that these notifications happen within a consistent soundscape in an aesthetically seamless way. We have implemented a prototype system using our approach and had very positive first results.

In order to fully verify it, we will conduct a user study with groups of two simultaneous users with different stimuli and try to verify that one user is effectively notified while the other one remains undisturbed. It will also be interesting to verify notification speed and effectiveness in comparison with regular (non-musical) notification sounds.

\section{ACKNOWLEDGMENTS}

This research was funded by Deutsche Forschungsgemeinschaft (DFG) within the FLUIDUM (Flexible User Interfaces for Distributed Ubiquitous Machinery) project.

\section{REFERENCES}

[1] M. Henz, S. Lauer, and D. Zimmermann. Compoze - intention-based music composition through constraint programming. In Proceedings of the 8th International Conference on Tools with Artificial Intelligence (ICTAI'96), page 118. IEEE Computer Society, 1996.

[2] F. Kilander and P. Lönnqvist. A whisper in the woods an ambient soundscape for peripheral awareness of remote processes. International Conference on Auditory Display, Kyoto/Japan, 2002.

[3] E. D. Mynatt, M. Back, R. Want, M. Baer, and J. B. Ellis. Designing audio aura. In Proceedings of the SIGCHI conference on Human factors in computing systems, pages 566-573. ACM Press/Addison-Wesley Publishing Co., 1998.

[4] C. Roads. The Computer Music Tutorial. MIT Press, 1996.

[5] M. Schmitz. SAFIR: A spatial audio framework for instrumented rooms. In Proceedings of ITI04: Workshop on Invisible and Transparent Interfaces, held at Advanced Visual Interfaces 2004, Gallipoli, Italy, 2004 . 